\title{
Volvulus of the transverse colon
}

\author{
N. J. McC. MORTENSEN \\ M.D., F.R.C.S.*
}

\author{
G. HoFFMAN \\ M.D., F.A.C.S. $\dagger$
}

Frenchay Hospital, Bristol

\begin{abstract}
Summary
Volvulus of the transverse colon is a rare condition requiring early diagnosis and treatment. In the absence of ischaemic changes within the redundant bowel, success can be anticipated with conservative fixation procedures. A case is reported which was treated by reduction, decompression and parallel colopexy. Although this disease has been infrequently reported in the past there may well be an increasing incidence with the introduction of high fibre diets and the use of the colonoscope.
\end{abstract}

\section{Introduction}

Volvulus of the transverse colon is an uncommon event. Although this segment of the colon is often quite mobile and redundant, its wide based mesenteric attachments prevent frequent torsion. Previous reports are largely limited to single cases although there are some collected series of as many as 18 cases (Kallio, 1932).

In this article, the authors report another case of volvulus of the transverse colon and review previous publications on the subject.

\section{Case report}

A 29-year-old asthenic female computer programmer was admitted as an emergency to Cossham Hospital. She gave a history of recurrent episodes of abdominal distension and colicky abdominal pain since childhood. For the 2 days before admission she had experienced another episode of colicky central abdominal pain, distension, copious vomiting and absolute constipation.

On examination she looked ill, was dehydrated and experienced distressing spasms of colicky abdominal pain. Her pulse was $80 / \mathrm{min}$ and regular, and she was normotensive. The apex beat was displaced to the right of her sternum and the heart sounds were maximal along the right parasternal edge. Her abdomen was grossly distended and tympanic, and there was a visible loop of dilated bowel beneath the right costal margin which was

*Present address: Southmead Hospital, Bristol.

$\nmid$ Present address: Norfolk Surgical Center, Norfolk, Virginia, U.S.A.

Reprint requests to: N. J. McC. Mortensen, Department of Surgery, Southmead Hospital, Bristol. slightly tender. Bowel sounds were obstructive Rectal and vaginal examinations were normal.

Plain chest and abdominal X-rays (Figs 1 and 2) revealed gross distension of the large bowel and elevation of the left hemidiaphragm. The cardiac shadow was shifted to the right.

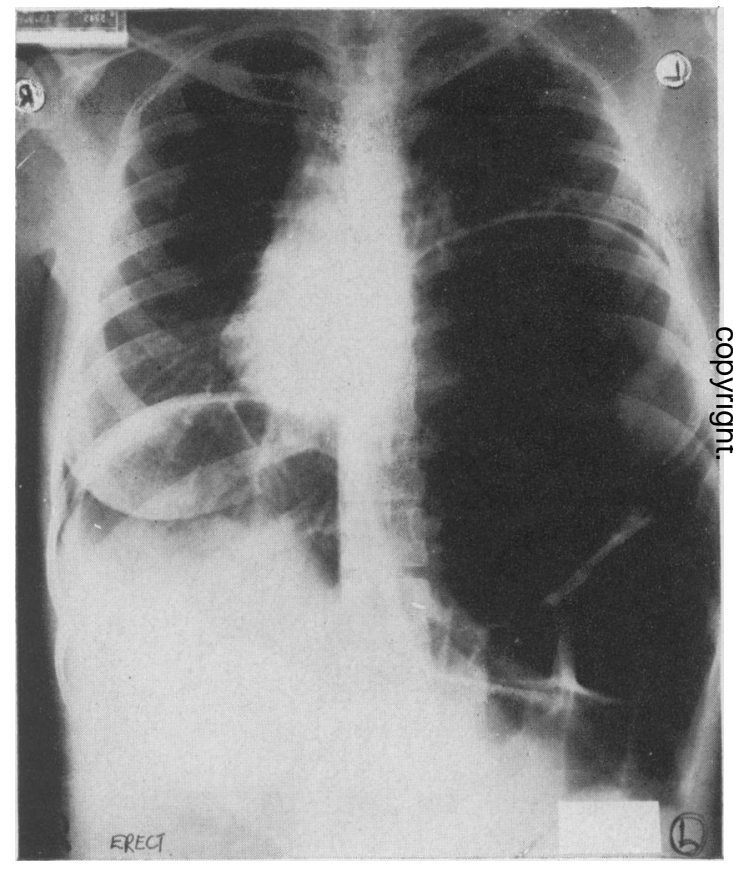

Fig. 1. Erect chest X-ray: Massive distension of the colon is apparent. The left hemidiaphragm is markedly elevated and the heart is shifted to the right. Another loop of distended bowel has come to lie beneath the right hemidiaphragm and displaced the liver inferiorly.

\section{Course}

At laparotomy a volvulus of the transverse colon was found with clockwise rotation through $180^{\circ}$ of the redundant colon. The affected bowel, suspended on a loose mesentery, was grossly dilated with gas, measuring $15 \mathrm{~cm}$ in diameter. Despite such a degree of dilatation the bowel wall, though thin, was not tense, suggesting an acute on chronic obstruction. 
The blood supply was unimpaired, and the colonic peritoneal reflections were normal. The caecum and asscending colon were moderately dilated. Descending and sigmoid colons were collapsed.

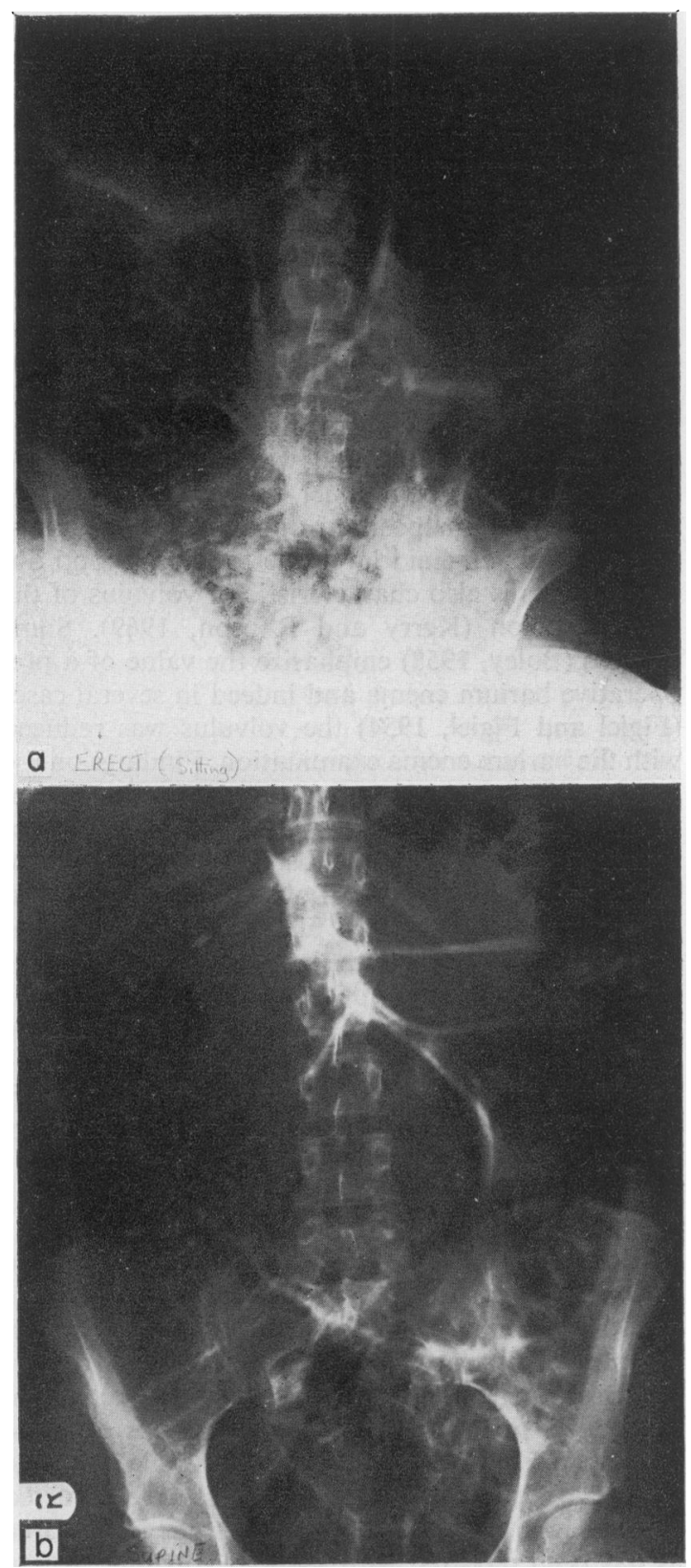

Fig. 2a and b. The distended loop of colon is seen to be in the upper abdomen. There is evidence of small bowel dilatation. The descending colon is of normal size. Overlying the third lumbar vertebra the distended loop can be seen to end in a 'beak' suggesting volvulus.
The volvulus was reduced, this being facilitated by aspiration of the intestinal gas with an 18-gauge needle passed obliquely through the colonic wall. The needle track was then closed with a chromic catgut suture. Since the long redundant loop of transverse colon was viable, a modified colopexy was performed. The loop of transverse colon hanging in a broad $U$-shape was sutured to the adjacent ascending and descending limbs of the colon with a continuous chromic catgut seromuscular suture on each side (Fig. 3).

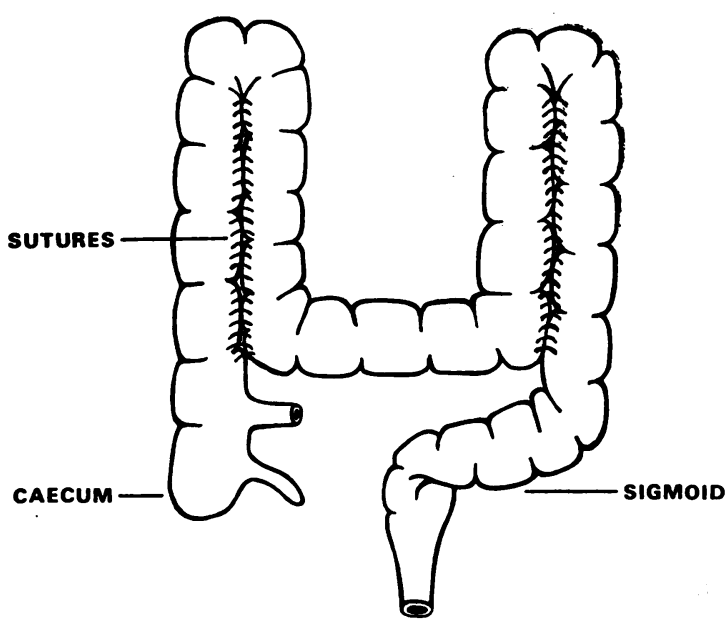

FIG. 3. Diagram to illustrate parallel colopexy after reduction and deflation.

Postoperatively the patient made a good recovery. Bowel sounds were present on the first postoperative day and by the third day she was tolerating a full diet. Nine months after surgery she remains well, and there have been no further episodes of abdominal pain. A barium enema 4 months postoperatively demonstrated the redundant loop in a good position with normal haustration and function.

\section{Discussion}

Volvulus of the colon is said to account for between $3 \%$ (Boley, 1958) and $10 \%$ of colonic obstructions (Kerry and Ransom, 1969). In a review of previous publications between 1961 and 1969, Kerry and Ransom (1969) reported 306 cases of colonic volvulus, of which only $4 \%$ involved the transverse colon. Such figures, however, do not take into account geographical variations in the incidence of this problem, and early reports (Kallio, 1932; Bruusgaard, 1947) suggest that transverse colon volvulus may account for up to $30 \%$ of all colonic volvuli in Eastern and Scandinavian countries.

\section{Aetiology}

The aetiology of transverse colon volvulus is 
probably a combination of primary anatomical factors and secondary contributory disease states. Gerwig (1955) has listed 4 anatomical variations which may increase the incidence of volvulus of the transverse colon:

1. Elongation of the mesentery with a freely movable bowel.

2. Absence of the mesentery with a mobile bowel. 3. Closely approximated points of fixation at the hepatic and splenic flexures.

4. Adhesions from previous surgery (Figiel and Figiel, 1954; Boley, 1958), or congenital bands (Weir and Wong, 1959).

To this can be added surgical defects in the small bowel mesentery through which the transverse colon might herniate and then twist. The most common anatomical abnormality appears to be the close approximation of the points of fixation of the transverse colon at the hepatic and splenic flexures with a long redundant transverse colon in between.

This anatomical variation may arise in several ways:

(i) as a result of a congenital problem with or without malrotation (Boley, 1958; Kerry and Ransom, 1969);

(ii) elongation of the transverse colon and its mesentery as a result of a high fibre diet (Singh and Kochber, 1970; Boley, 1958) - this is said to be the reason for the high prevalence of the condition in India and Eastern Europe, or conversely as a consequence of chronic constipation in elderly subjects (Boley, 1958; Kerry and Ransom, 1969);

(iii) it may arise in an acute situation with overdistension of the transverse colon as may occur in acute distal obstruction (Boley, 1958, Kerry and Ransom, 1969) or following the insufflation of air during colonoscopy (Britton et al., 1977).

A number of secondary contributing factors have also been described. Lesions within the colonic wall such as submucosal hamartomas (Lapin et al., 1973) háve been implicated. Malreposition of the colon after surgery (Zaslow and Orloff, 1954; Cope, 1968) has been described, and the condition has also presented in the post partum period (Murray, 1959). Single cases have been described in association with organo axial volvulus of the stomach (Jones and Jopes, 1972), after trauma (Massot and Laine, 1965), and in a patient with scleroderma (Budd et al., 1977).

\section{Clinical}

The clinical features are those of any acute volvolus of the colon, including the sudden onset of colicky abdominal pain, distension, absolute constipation and at a later stage, vomiting. As illustrated in this case report a significant number of patients have a history of recurrent episodes of pain and abdominal distension, relieved by a bowel motion. These probably represent attacks of volvulus followed by spontaneous reduction before the episode eventually resulting in surgery (Martin and Ward, 1944; Figiel and Figiel, 1954; McGowan, Soriano and McCausland, 1957; Kerry and Ransom, 1969). The 4 most common physical signs in all cases of volvulus are abdominal distension, a visible or palpable abdominal mass, shock and pyrexia (Kerry and Ransom, 1969). In transverse colon volvulus this mass is most often located in the right upper quadrant of the abdomen. As time elapses signs of vascular insufficiency of the involved loop may become apparent.

The diagnosis of this uncommon condition depends upon radiological investigation (Figiel and Figiel, 1954; Boley, 1958; Kerry and Ransom, 1969). Typical findings on plain films include massive dilatation of the colon and with loops having the appearance of a 'bent inner tube'. There may be redundancy or malposition apparent on the films, and a double air-fluid level due to 'closed loop obstruction' as is also characteristic of volvulus of the sigmoid colon (Kerry and Ranson, 1969). Some authors (Boley, 1958) emphasize the value of a preoperative barium enema and indeed in several cases (Figiel and Figiel, 1954) the volvulus was reduced with the barium enema examination. Findings on the barium enema include: funnel-shaped obstruction in the proximal half of the transverse colon, or round termination of the barium column with a dentate margin at the site of obstruction in the distal transverse colon. Detailed films at the level of obstruction may show spiralling and crossing of the mucosal folds at that site. Other authors feel that delay in surgical treatment in order to perform the barium enema is not warranted, and that indeed the procedure may not only be time-consuming but it might also cause perforation (Kerry and Ransom, 1969).

\section{Treatment}

Although conservative measures may be useful in the treatment of sigmoid volvulus, most authors would agree that laparotomy in transverse colon volvulus is mandatory. There is, however, considerable disagreement as to the correct definitive surgical procedure. In the presence of gangrenous or questionably viable bowel a resection is indicated. The hazards of a primary anastomosis of obstructed bowel might be expected to be the same in this area as has been reported with sigmoid volvulus. Where the involved colon is viable a more conservative procedure is indicated. Some are content with simple reduction (Singh and Kochber, 1970) but there is a considerable risk of recurrence, indeed, as already noted, many of these patients present with a history 
suggesting previous volvulus of the transverse colon with spontaneous reduction. Primary simple reduction followed by a secondary resection might be considered a safer procedure. Fixation of the redundant loop of transverse colon to the anterior abdominal wall (McGowan et al., 1957; Jones and Jones, 1972) seems to be a simple and effective procedure and has been combined with decompression of the transverse colon using an indwelling De Pezzer catheter (Zaslow and Orloff, 1954). The present authors report a further alternative to resection-reduction and decompression followed by parallel colopexy (Fig. 3) - which has had a satisfactory outcome. Unfortunately, there are not enough previously recorded cases to make any definitive statements about the surgical procedure of choice. It is, however, safe to say that many cases have been successfully treated in the absence of ischaemia with conservative fixation procedures rather than resection. Both Kerry and Ransom (1969) as well as Wangensteen (1955) reported a $33 \%$ mortality in the treatment of volvulus of the transverse colon. Whether this reflects a delay in surgery, poor general condition of the patients, or the complications of radical surgery is not clear.

\section{Acknowledgments}

We should like to thank Mr R. E. May for permission to report this case, and Miss J. Masterman for typing the manuscript.

\section{References}

Boley, S.J. (1958) Volvulus of the transverse colon. American Journal of Surgery, 96, 122.

Britton, D.C., Tregoning, D., Bone, G. \& McKelvey,
S.T.D. (1977) Colonoscopy in surgical practice. British Medical Journal, 1, 149

BruUsgaARd, C. (1947) Volvulus of the sigmoid colon and its treatment. Surgery, 22, 466.

Budd, D.C., Nirdlinger, E.L., Sturtz, D.L. \& Fouty, W.J. (1977) Transverse colon volvulus associated with scleroderma. American Journal of Surgery, 133, 370.

COPE, Z. (1968) In: The Early Diagnosis of the Acute Abdomen. 13th edn, p. 122. Oxford University Press, London.

Figiel, L.S., Figiel, S.J. (1954) Volvulus of the transverse colon. Radiology. St. Paul, etc., 63, 832.

GERWIG JR., W.H. (1955) Volvulus of the colon. Surgical Clinics of North America, 35, 1395.

JONES, W.M. \& JONES, C.D.P. (1972) Volvulus of the transverse colon associated with organo-axial volvulus of the stomach. American Journal of Surgery, 124, 404.

Kallio, K.E. (1932) Über Volvulus coli transversi. Acta chirurgica scandinavica, 70, 39.

KERRY, R.L. \& RANSOM, H.K. (1969) Volvulus of the colon. Archives of Surgery, 99, 215-221.

Lapin, R., Kane, A.A., LeE, C.S. \& Rifat, H. (1973) Volvulus of the transverse colon associated with submucosal hamartomas. American Journal of Gastroenterology. New York, 59, 170.

McGowan, J.M., Soriano, S. \& McCausland, W. (1957) Volvulus of the transverse colon. American Journal of Surgery, 93, 857.

MARTIN, J.D. \& WARD, C.S. (1944) Megacolon associated with volvulus of the transverse colon. American Journal of Surgery, 64, 412.

Massot, P. \& LAINE, J.L. (1965) Volvulus du colon transverse chez l'enfant. Annales de chirurgie infantile. Paris, 6, 145.

Murray, A. (1959) Volvulus of the transverse colon complicating labour. British Medical Journal, 2, 659.

Singh, A. \& Kochber K.S. (1970) Volvulus of the transverse colon. Diseases of the Colon and Rectum, 13, 397.

Wangensteen, O.H. (1955) In: Intestinal Obstructions, 3rd edn, p. 661. Charles C. Thomas, Springfield.

WEIR, D.C. \& WONG, J.C. (1959) Volvulus of the transverse colon due to congenital bands. Missouri Medicine, 56, 908.

Zaslow, J. \& ORLOFF, T. (1954) Volvulus of the transverse colon occurring as a postoperative complication. American Journal of Surgery, 87, 780. 